\title{
A Smartphone-Based Personalized Activity Recommender System for Patients with Depression
}

\author{
Galen Chin-Lun Hung * \\ Department of Addiction \\ Science, Taipei City Psychiatric \\ Center, Taipei City Hospital, \\ Taipei, Taiwan \\ galenhung@tpech.gov.tw
}

\author{
Pei-Ching Yang \\ Department of Computer \\ Science and Information \\ Engineering, National Cheng \\ Kung University, Tainan, \\ Taiwan \\ yang.peiching@iir.csie.ncku.edu.tw
}

\author{
Chen-Yi Wang \\ Institute of Medical Informatics \\ National Cheng Kung \\ University, Tainan, \\ Taiwan \\ chen_yi@iir.csie.ncku.edu.tw
}

\author{
Jung-Hsien Chiang* \\ Department of Computer \\ Science and Information \\ Engineering, National Cheng \\ Kung University, Tainan, \\ Taiwan \\ jchiang@mail.ncku.edu.tw
}

\begin{abstract}
Depression is a common mental illness worldwide. Apart of pharmacological treatment and psychotherapy, self-management of negative emotions is of paramount importance, because relapse of depression often results from an inadequate response to negative emotions. The purpose of this study is to design and implement a personal recommender system, for emotion regulation. It assists users to be aware of negative emotions and guides them to deal with it with behavioral activation. It analyzes the smartphone usage patterns to predict the emergence of negative emotions, while integrating data obtained from context awareness and psychiatrists' recommendations to suggest relevant emotion-regulating activities. The presented system has a potential to provide personalized and pervasive mental health services for patients with depression.
\end{abstract}

\section{Keywords}

Depression; metal health; usage patterns; emotion; context-aware; recommender

\section{INTRODUCTION}

Depression is a common illness worldwide, according to the World Health Organization, with an estimated 350 million people affected by depression in various degrees of severity [1]. Apart of pharmacological treatment and psychotherapy, self-management of negative emotions is of paramount importance, because relapse of depression often results from an inadequate response to negative emotions. In addition to those with clinical depression, more and more healthy individuals are beginning to recognize how negative emotions may impede their performance, relationship, and quality of life. Therefore, demand is rising for the ability to promptly aware and manage one's negative emotions [2-5].

Finding a personal activity recommender system who offers seamless and continuous guidance for emotion recognition and regulation is desirable, but often difficult. Traditional psychotherapy or coaching usually limits to a number of sessions in a treatment setting. Ideally, one would like to measure emotion and its behavioral correlates in real time to offer context-specific suggestions [6]. The popularity of smartphone has provided ample opportunities for a personalized activity recommender system for regulating emotions. The analysis of smartphone user behaviors is beginning to gain traction. This behavioral pattern can be either the cause or the consequence of human emotion. Researchers are beginning to examine the association between the smartphone usage pattern and the concurrent emotion, aiming at monitoring and predicting emotions automatically, followed by context-specific recommendations. There are a significant number of mobile applications (apps) available for the smartphone that track emotions. The majority of applications are designed to help users record their moods (e.g., MoopScope, Mood Panda) [7-13]. Only a piecemeal of research tries to predict users' emotional state based on smartphone usage patterns [12,13].

In this paper, we provide new insights into mobile computing applications for emotion detection via mobile phone usage patterns, and the use of context-awareness combined with a recommendation database to suggest feasible activities to relieve negative emotions. We also propose the use of "easy to understand and select" candidate items in the mobile environment, making it very easy for users of any age to understand if a suggestion item is in order by their action event history or activities engaged by similar users.

The purpose of this study is to establish a personal emotion-regulating recommender system based on mobile phone usage patterns to assist users be aware of negative emotions and to help learn how to contain and change them.

The specific aims of this research are:

a. To record negative emotions in a daily basis and build a negative emotions classification model;

b. To extract significant feature sets from smartphone usage patterns;

c. To establish the emotion-regulating action knowledge based on expert recommendations;

d. To develop a personal emotion-regulating recommender system.

Through the above steps, we develop, design and build a personal emotion-regulating recommender system. These technologies not only recognize, analyze, and quantify users' negative emotions, but also remind them and make recommendations, all of which create new values of a wireless sensor network in mental health applications. 


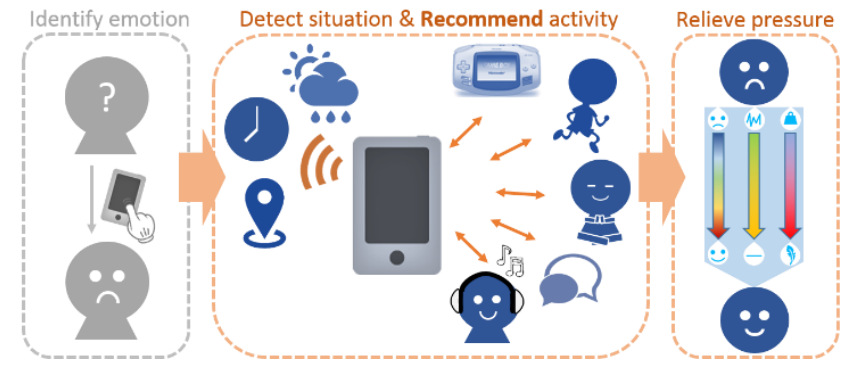

Figure 1. The concept of smartphone-based personalized activity recommender system

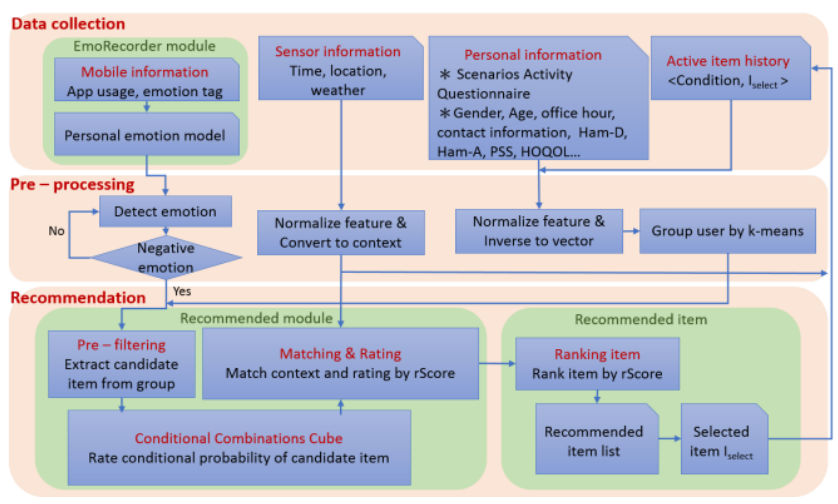

Figure 2. System flow diagram

\section{MATERIALS AND METHODS}

Figure 1 shows the concept of our system. We identified negative emotions gathered by the EmoRecorder module [14] that analyzed users' application usage on smartphone to detect emotion states. When negative emotion was identified, the system detected current environmental contexts to make an appropriate activities recommendation.

The system flow diagram includes three parts: 1). Data collection, 2). Pre-processing, and 3). Recommendation, as shown in Figure 2. We describe the details in the following sections.

\subsection{Data Collection}

We used four components on the android smartphone to collect data: EmoRecorder module, personal information, sensor information, and item history. Content of components are summery in Table 1.

If the participants used our system at the first time, we asked them to fill out a questionnaire on the smartphone that collected their personal information, such as age, gender, occupation, office hours, and so on. They also completed a scenario activity questionnaire that requested the participants to select the activities of interest under different context. In this paper, we defined a context as a combination of environmental factors (e.g. Office hour, workplace, weather). Each recommended activity was used as an item in our recommender system. We used EmoRecorder module, our previous work, to process users' application usage and emotion tags that detected users' emotion states. The emotion states were labeled according to three dimensions of participant's negative emotions: depression, anxiety, and stress. Each dimension was further divided into three levels: low, middle, and high.
Table 1 Materials of recommender system

\begin{tabular}{|c|c|c|}
\hline Type & Category & Content \\
\hline \multirow{2}{*}{$\begin{array}{l}\text { EmoRecorder } \\
\text { module }\end{array}$} & $\begin{array}{c}\text { Mobile } \\
\text { information }\end{array}$ & $\begin{array}{l}\text { \{app usage, depression level, } \\
\text { anxiety level, stress level }\}\end{array}$ \\
\hline & $\begin{array}{c}\text { Personal } \\
\text { emotion model }\end{array}$ & $\begin{array}{l}\text { \{depression level, anxiety } \\
\text { level, stress level }\}\end{array}$ \\
\hline \multirow[b]{2}{*}{$\begin{array}{l}\text { Personal } \\
\text { information }\end{array}$} & Personal profile & $\begin{array}{l}\text { \{age, gender, occupation, } \\
\text { office hour, favorite activity }\end{array}$ \\
\hline & $\begin{array}{c}\text { Scenarios } \\
\text { activity } \\
\text { questionnaire }\end{array}$ & $\begin{array}{l}\text { \{time, location, weather, } \\
\text { active item }\}\end{array}$ \\
\hline \multirow{3}{*}{$\begin{array}{l}\text { Sensor } \\
\text { information } \\
(\text { context })\end{array}$} & Time & $\begin{array}{l}\text { \{office hour, non-office } \\
\text { hour }\end{array}$ \\
\hline & Location & $\begin{array}{l}\text { \{company, home, outside, } \\
\text { inside\} }\end{array}$ \\
\hline & Weather & $\begin{array}{l}\text { \{uncomfortable, } \\
\text { comfortable }\}\end{array}$ \\
\hline \multirow{8}{*}{$\begin{array}{l}\text { Active item } \\
\text { history } \\
\text { (item) }\end{array}$} & Cognitive game & $\begin{array}{l}\text { \{reaction game, color game, } \\
\text { trail making test }\}\end{array}$ \\
\hline & $\begin{array}{l}\text { Mindfulness } \\
\text { practice }\end{array}$ & $\begin{array}{l}\text { \{ sitting meditation, stretch } \\
\text { meditation, brief meditation }\}\end{array}$ \\
\hline & Sport & $\begin{array}{l}\text { \{cardiovascular sport, } \\
\text { strength and endurance sport, } \\
\text { flexibility sport, ball sport }\}\end{array}$ \\
\hline & Music & $\{$ Crystal music on YouTube $\}$ \\
\hline & Movie & $\{$ Micro Movie on YouTube $\}$ \\
\hline & Reading & $\{$ Mind Books $\}$ \\
\hline & Communication & $\begin{array}{l}\text { \{Chatting with friend and } \\
\text { family }\}\end{array}$ \\
\hline & Food & $\{$ Nearby cuisine $\}$ \\
\hline
\end{tabular}

When we recommended an activity, we detected users' current location using GPS (or Wi-Fi) and time to determine their environmental context. We obtained location's current weather by api.openweathermap.

Activity item history recorded users' current context and the chosen activities (e.g. Office hour, workplace, comfortable weather, music).

\subsection{Preprocessing}

In this phase, there are two processes. First, once the system identified negative emotion, it triggered the recommendation. We defined negative emotions as depression or anxiety in the high level or depression and anxiety both in the middle level (see Figure 3). Second, the personal information and activity item history were converted into a vector representation. Categorical data were converted into binary values while numerical data were normalized to [0..1]. Each participant had a unique personal vector. We grouped the users using a clustering method, k-means. The reason for grouping was that similar users may prefer similar activities. Therefore, we recommended activities that similar users have ever performed. Furthermore, we applied the Davies-Bouldin index (DB index) [15] to evaluate cluster validity in the recommendation algorithm. The Davies-Bouldin indexis the ratio of the sum of the within cluster scatter to the between cluster separation. A lower DB index means a better result of clustering. 


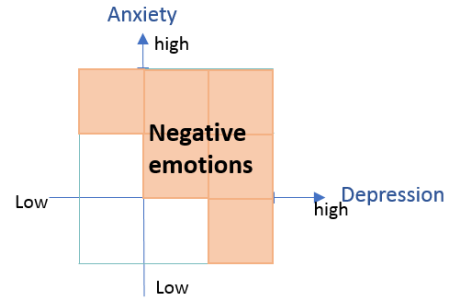

Figure 3 Negative emtions identification

\subsection{Recommendation}

In the recommended module, the system collected users' activity of the same group that has been implemented as the candidate item set.

$$
S=\left\{\text { item }_{u} \mid u \in \text { user group }\right\}
$$

Furthermore, we assume that the item rating was affected by three factors: item, user, and context. The item rating can be stored in a three-dimensional cube, such as the one shown in Figure 4. We entitle this cube a conditional combinations cube (CCC). Each candidate item was represented as a conditional combinations matrix (CCX) with different contexts. Under a specific context, a candidate item was expressed as an array. Each element of the array is the conditional probability $I_{k}\left(u_{n}, c_{j}\right)$ that the user $n$ chosen the item $k$ under the context $j$. In this paper, we calculated the conditional probability from active item history. Users of the same group has the same candidate item set $S$ they shared a common Conditional Combinations Cube.

Take Figure $4 \mathrm{a}$ for an example: The recommended activity (item) was "running". The corresponding context $c_{1}$ was [non-office hour, home, comfortable weather]. The probability that the user 1 will "running" under the context of non-office hour, home, and comfortable weather was 0.8 . Similarly, the probability of user 2 will "running" was 0.65 .

When the participant's negative emotion was recognized, the system subsequently detected the present context and requests all the item arrays that matched the present context from CCC. For example in Figure $4 \mathrm{~b}$, if the system detects the context $c_{1}$ and then the item arrays with context $c_{l}$ were acquired from the CCC.

The candidate items were rated according to a ranking score function, $r \operatorname{Score}\left(I_{k}\right)$. rScore $\left(I_{k}\right)$ combined probabilities of the target user and the other users in the same group. It is shown as follows:

$\operatorname{rScore}\left(I_{k}\right)=\frac{I_{k}\left(u_{n}, c_{j}\right)+\alpha \times I_{k}\left(u_{\text {group }}, c_{j}\right)}{2}$

where $I_{k}\left(u_{n}, c_{j}\right)$ means probability of the target user $n$ perform item $k$ under context $j, \quad I_{k}\left(u_{\text {group }}, c_{j}\right)$ means probability of the other users in the same group perform item $k$ under the same context $j$. If $m$ is the number of users in user group, $I_{k}\left(u_{\text {group }}, c_{j}\right)$ is calculated as follows:

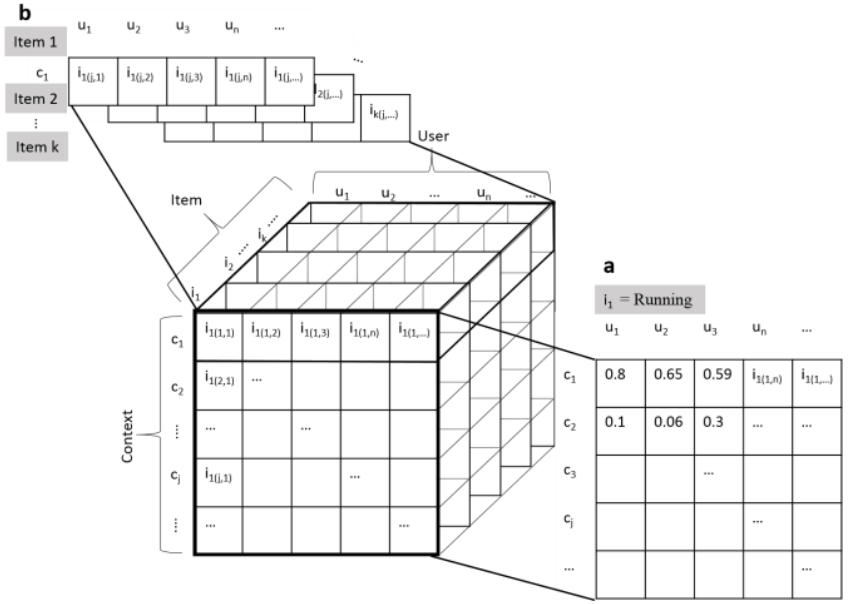

Figure 4 Conditional Combinations Cube

$$
I_{k}\left(u_{\text {group }}, c_{j}\right)=\frac{\sum_{i=1}^{m} I_{k}\left(u_{n}, c_{j}\right)}{m}, m \neq n
$$

$\alpha$ is weight parameter and is defined as follows:

$$
\alpha=\left\{\begin{array}{c}
1, \text { if } \mathrm{DB} \leq 0 \\
\frac{1}{\mathrm{DB}^{2}}, \text { if } \mathrm{DB}>0
\end{array}, 0 \leq \alpha \leq 1\right.
$$

Finally, the system recommends item list to the user. The system also records the history of user interaction with the items. The participant's historical data contains the present context and the items selected by the participant from the recommendation list.

\section{EXPERIMENTAL DESIGN}

In this session, we introduce the design of an experiment intended to evaluate the performance of our recommender system.

\subsection{Design Process}

Figure 5 shows the flowchart of the experiment. The screenshots of the activity recommender system on mobile in Figure 6. We recruited subjects to join the experiment and assisted them downloading our recommender system to their own Android smartphones. Subjects were asked to fill out questionnaire that included personal information and scenario activity questionnaire. The system automatically detected users' emotion states in background process by EmoRecorder module using general emotion model after the questionnaires were completed. The system made context-specific activity recommendation list for user whenever negative emotion was identified. Users had to choose an activity that they want to do and tag their emotion states (depression, anxiety, and stress) after completion of the suggested activity.

We planned to compare our approach with the following methods:

Method I: Random (baseline)

Method II: CCC (all user as the same group);

Method III: Clustering + CCC (ours);

We used the mean average position $(M A P)$ and the mean absolute error $(M A E)$ to evaluate our approach and other approaches. MAP indicates whether the recommender system conforms to user's intent. A larger MAP value represents a better performance. MAP is calculated as follows: 


$$
M A P=\frac{1}{n} \sum_{i=1}^{n} \frac{1}{p_{i}}
$$

where $n$ denotes the number of recommendation, pi denotes $p$-th position on the recommendation list.

$M A E$ measures the deviation between the true rating and the predicted rating of the relaxing activity. The smaller MAE value represents a better performance. MAE is calculated as follows:

$$
M A E=\frac{1}{n} \sum_{i=1}^{n}\left|p_{i}-\alpha_{i}\right|
$$

where $\mathrm{n}$ denotes the number of recommended items, $p_{i}$ is the $r S$ core value of the selected item, and $\alpha_{i}$ is the $r$ Score value of the top one item.

\subsection{Participants}

In this pilot study, we recruited 15 normal subjects with Android smartphone and asked them to use our recommender application for 14 days. Only 5 participants remained active use at the end.

The recruitment of normal subjects in the first stage avoids potential difficulties for smartphone usage among profoundly depressed patients, while increasing the generalizability of our findings to recovered patients and the general public.

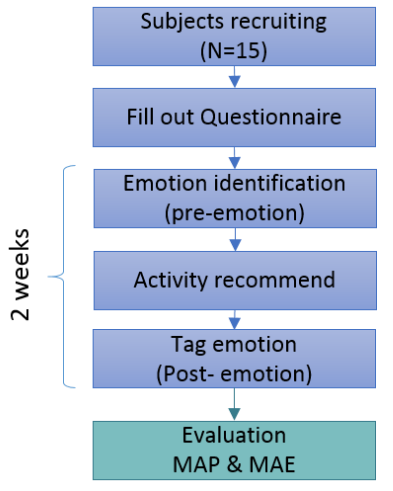

Figure 5 Process of the experiment

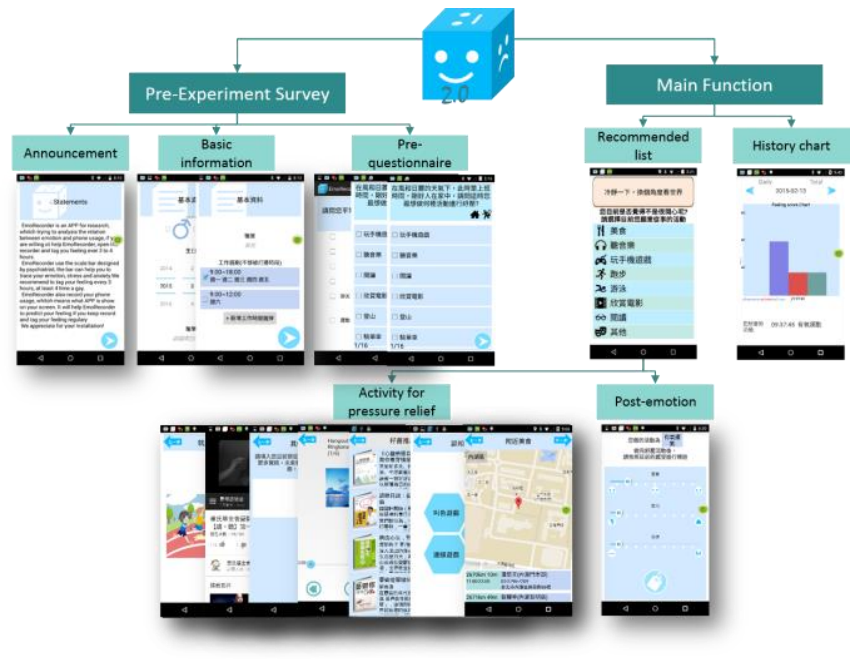

Figure 6 Overview of recommender system

\subsection{Experimental results}

To evaluate our proposed method, we collected valid data that the recommended activity exactly corresponds to an emotion state tagged.

Figure 7 presents the mean average position by days of active use. The result showed that the proposed approach CCC and clustering + CCC were both better than baseline (random). The average $M A P$ of methods was $0.18,0.38$, and 0.39 . We utilized Mann-Whitney U test and observed that method II and method III were both significantly superior than method I ( $p$-value $<0.0001$ ); method II and method III were not performing differently $(p$-value $=0.887)$. It appeared that clustering didn't change the performance of our recommender system. Figure 8 shows the mean average error by number of days to use recommender system. MAE meant the actual difference between our system and users' preference. The smaller, the better. Apparently, our precision of suggestion improved from baseline. The average $M A E$ of methods is $0.22,0.13$, and 0.07 . We implemented pair T-test and observed that method II and method III were both significantly superior than method I (p-value were 0.004 and $<0.001$ ); method III outperformed method II (p-value <0.0001). On the contrary, clustering improved the performance of recommender system dramatically.

In order to verify that whether our recommender system effectively alleviated users' negative emotions, we implemented Wilcoxon Signed Rank test to examine differences between emotional tags before and after a suggested activity. The total number of emotion tags (depression, anxiety, and stress) was 211. The result of Wilcoxon test of two-tail was significant $(\mathrm{p}<0.0001)$.

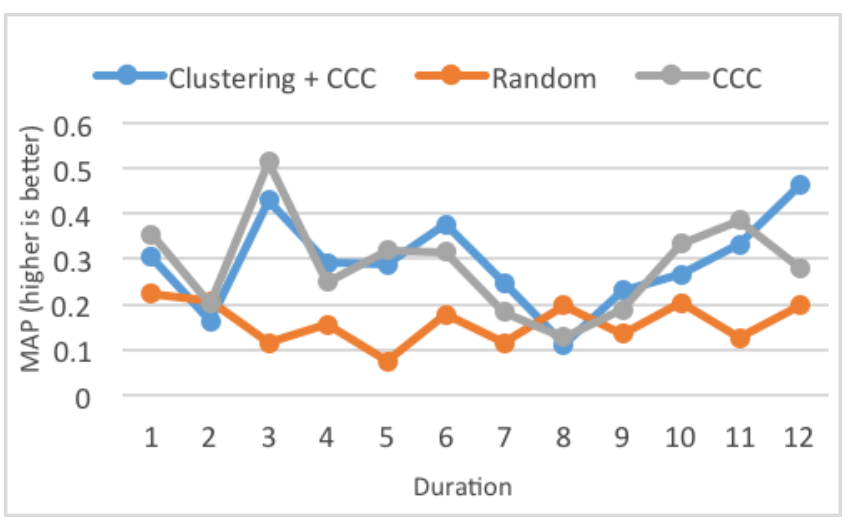

Figure 7 Mean Average Position (MAP)

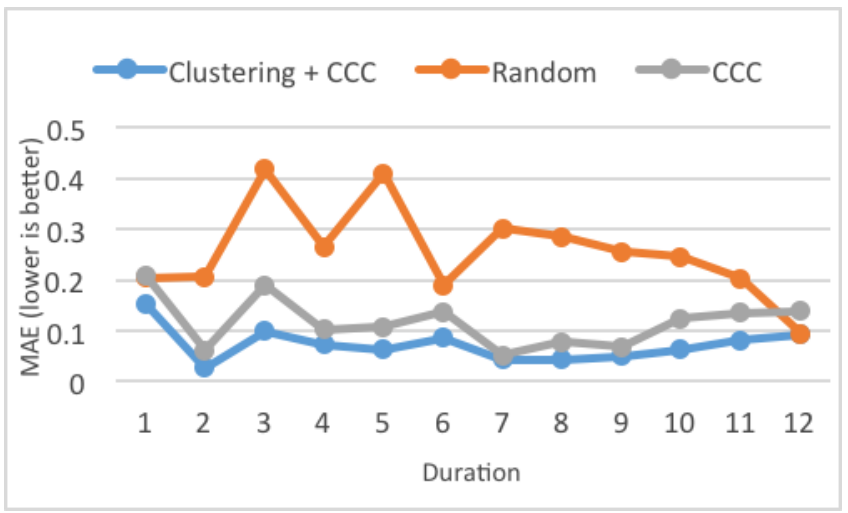

Figure 8 Mean Average Error (MAE) 
The results of our experiment verified two facts. One was that the precision of our recommendation improved with time, and the other was that our personal activity recommender system did help users alleviate negative emotions.

\section{CONCLUSIONS AND FUTURE WORK}

In this paper, we developed a smartphone-based personalized activity recommender system on Android smartphones for the recognition and self-management of negative emotions. Our recommender system considers the user's and similar subjects' preferences of activity and histories of choices simultaneously in the current context to make the best recommendations. In this pilot study, we verified that our proposed recommender system was able to provide useful suggestions, which indeed alleviated users' negative emotions. In this study, we presented the performance of our system in normal subjects, an examination of this system among patients with clinical depression is being carried out currently.

Our future work includes the analysis of the data obtained from patients with clinical depression to examine its effectiveness. We would like to test our proposed recommender system among 30 outpatients with depression for 8 weeks and evaluate if precision of recommender system will improve continuously. Moreover, we would like to make our system capable of communicating with the electronic health record, which would further the detection of clinically notable emotional alteration. We plan to make our results publicly available so mobile systems developers targeting depressed patients can use our findings as the stepping stone to facilitate their works, which is crucial in improving the conditions of public mental health.

\section{ACKNOWLEDGMENT}

We thank the participants \& students involved in the study.

\section{REFERENCES}

[1] Organization WH. Depression. Secondary Depression 2012. http://www.who.int/mediacentre/factsheets/fs369/en/. Accessed on July 2014.

[2] Foundation MH. Mental Health Statistics. Secondary Mental Health Statistics n. d. http://www.mentalhealth.org.uk/help-information/mental-hea lth-statistics/. Accessed on July 2014.

[3] Center WDH. How Stress, Anxiety, and Depression Affect Your Health. Secondary How Stress, Anxiety, and Depression Affect Your Health 2014. http://www.webmd.com/depression/stress-anxiety-depression. Accessed on July 2014.

[4] HELPGUIDE.org. Depression Symptoms \& Warning Signs. Secondary Depression Symptoms \& Warning Signs. http://www.helpguide.org/mental/depression_signs_types_di agnosis_treatment.htm. Accessed on September 2014.

[5] Pyszczynski, T., and Greenberg, J. 1987. Self-regulatory perseveration and the depressive self-focusing style: a self-awareness theory of reactive depression. Psychological bulletin, 102(1), 122.

[6] Jean, F. A., Swendsen, J. D., Sibon, I., Fehér, K., \& Husky, M. 2013. Daily Life Behaviors and Depression Risk Following Stroke A Preliminary Study Using Ecological Momentary Assessment. Journal of geriatric psychiatry and neurology, 26(3), 138-143.

[7] LiKamWa, R., Liu, Y., Lane, N. D., \& Zhong, L. 2011. Can your smartphone infer your mood? In PhoneSense workshop (pp. 1-5).

[8] LiKamWa, R., Liu, Y., Lane, N. D., and Zhong, L. 2013. Moodscope: building a mood sensor from smartphone usage patterns. In Proceeding of the 11th annual international conference on Mobile systems, applications, and services (pp. 389-402). ACM.

[9] Mood Panda. http://www.moodpanda.com. Accessed on October 2014.

[10] Kramer, I., Simons, C. J., Hartmann, J. A., Menne-Lothmann, C., Viechtbauer, W., Peeters, F., Wichers, M. 2014. A therapeutic application of the experience sampling method in the treatment of depression: a randomized controlled trial. World Psychiatry, 13(1), 68-77.

[11] Faurholt-Jepsen, M., Frost, M., Vinberg, M., Christensen, E. M., Bardram, J. E., \& Kessing, L. V. 2014. Smartphone data as objective measures of bipolar disorder symptoms. Psychiatry research, 217 (1), 124-127.

[12] Grunerbl, A., Muaremi, A., Osmani, V., Bahle, G., Ohler, S., Troster, G., Lukowicz, P. 2015. Smartphone-based recognition of States and state changes in bipolar disorder patients. Biomedical and Health Informatics, IEEE Journal of, 19(1), 140-148.

[13] Huang, S. H., LePendu, P., Iyer, S. V., Tai-Seale, M., Carrell, D., \& Shah, N. H. 2014. Toward personalizing treatment for depression: predicting diagnosis and severity. Journal of the American Medical Informatics Association, 21(6), 1069-1075.

[14] Hung, G. C. L., Yang, P. C., Chang, C. C., and Chiang, J. H. 2014. Machine Learning-Based Analysis of Smartphone Usage Pattern and Its Association with Negative Human Emotion. Wireless Health 2014, Bethesda, MD.

[15] Davies, D. L., and Bouldin, D. W. 1979. A cluster separation measure. Pattern Analysis and Machine Intelligence, IEEE Transactions on, (2), 224-227. 